\title{
Treatment of Abdominal Aortic Aneurysms with Accompanied Iliac Artery Aneurysms Using New Sack Sealing Device
}

\author{
Kaspars Kisis *, ****, Janis Savlovskis **, ***, Polina Dombure *, ****, Marcis Gedins *, \\ Natalija Ezite **, ****, Kristaps Zarins ${ }^{* * * *}$, Dainis Krievins *, *** \\ * Department of Vascular Surgery, Pauls Stradins Clinical University Hospital, Riga, Latvia \\ ** Department of Radiology, Pauls Stradins Clinical University Hospital, Riga, Latvia \\ $* * *$ University of Latvia, Riga, Latvia \\ **** Riga Stradins University, Riga, Latvia \\ ***** Stanford University, Palo Alto, USA
}

\section{Summary}

Introduction. $20-30 \%$ of abdominal aortic aneurysms (AAA) occur simultaneously with unilateral or bilateral common iliac artery aneurysms (CIAA). Endovascular aneurysm repair (EVAR) is known to be an effective AAA treatment method used by many centres in over $80 \%$ of cases. Presence of AAA accompanied by CIAA significantly increases the complexity of EVAR with currently available endografts, internal iliac artery (IIA) often requiring coil embolisation resulting in serious post-procedural complications such as ischaemia of pelvic organs, gluteal claudication and erectile dysfunction.

Aim of the study. Demonstrate successful endovascular AAA and CIAA treatment with new generation sac-sealing endograft device. Materials and methods. From 2008 Pauls Stradins Clinical University Hospital is participating in the prospective clinical trial assessing the efficacy and stability of the new generation sac-sealing endograft device (Nellix®, Endologix, USA). Until now this trial had 40 enrolled patients with suitable for endovascular treatment aneurysmal morphology. The treatment group has included 16 patients with AAA extending to either one or both common iliac arteries (CIA). The control group consisted of 24 patients with isolated AAA. AAA diameter was $5.6 \pm 0.76 \mathrm{~cm}(\min -4.3$, max -6.98$)$ and $5.16 \pm 0.91 \mathrm{~cm}(\min -3.78, \max -7.24)$ in the treatment and control groups respectively. Seven patients had unilateral and nine patients had bilateral CIAA. The diameter of CIAA was $2.61 \pm 0.57$ $\mathrm{cm}$ (min - 2.04, max -4.44). Post-procedural follow-up was done at one, six and twelve months and on annual basis thereafter During follow-up the general health condition of the patients was assessed as well as computer tomography angiography (CTA) and duplex ultrasonography (DUS) imaging was performed in order to examine the status of the aneurysm, endograft condition and patency of IIA. Statistical analysis of data was performed using v19.0 SPSS software (IBM).

Results. All patients successfully treated with new generation sac-sealing endograft excluding AAA and CIAA from blood circulation. Average follow-up period was 18 months. Upon follow-up in both groups endograft was stable and fixated in aneurysms with no endoleaks detected. In the treatment group all treated IIAs had remained patent with no pelvic organs ischaemia or gluteal claudication symptoms.

Conclusion. New generation sac-sealing endograft is effective and simple in employment for the treatment of concomitant AAA and CIAA allowing the treatment of aneurysms with complex morphology and preserving the blood flow to internal iliac arteries. Further studies are required for long-term assessment of this endograft efficacy.

Key words: internal iliac arteries; abdominal aortic aneurysm; endovascular aneurysm repair; aneurysm sac sealing device; Nellix device.

\section{INTRODUCTION}

Abdominal aortic aneurysm (AAA) is defined as an increase in aortic diameter larger than $3 \mathrm{~cm}$ and its prevalence in population aged between 59 to 79 years is $5.9 \%(18)$. In the event of untreated AAA the most common adverse event is aneurysmal rupture with mortality exceeding $75 \%$. Even if the patient is admitted to hospital with ruptured AAA and treated by either open or endovascular repair, the associated perioperative mortality remains very high exceeding $50 \%$ (18). Therefore timely detection and elective AAA reconstruction is extremely important. Currently AAA reconstruction is possible using two methods: open and endovascular aneurysm repair (EVAR) (9, 18, 14, 23, 25). In the event of open repair AAA is resected and replaced with synthetic graft, whereas in the event of
EVAR a self-expanding endograft is implanted into aorta under x-ray control with fixation being made in the relatively unchanged aortic segments proximally from AAA and distally in non-aneurysmal iliac arteries.

In $20-30 \%$ of cases AAA continues to either one or both common iliac arteries (CIA) with CIA diameter exceeding $20 \mathrm{~mm}(18,27)$ (Figure 1). Currently available devices for AAA endovascular repair require unchanged iliac artery anatomy for distal fixation. If CIA is aneurysmatic then fixation is made in external iliac artery (EIA). In order to avoid retrograde filling of CIAA (type 2 endoleak) and prevent possible rupture, the internal iliac artery (IIA) must be occluded using coil embolisaiton or special occluders (2). Occlusion of IIA (and more importantly bilateral occlusion of IIAs) may result in important post-procedural complications 
such as spinal cord and intestinal ischemia, impotence, gluteal claudication and necrosis of gluteal and/ or perineal tissues $(3,6,10,21,22,24,26,28)$. The consequences of IIA occlusion depend on the blood supply requirements of pelvic organs that is usually more intensive in younger patients and patients with reduced cardiac output (7). The incidence of various complications after IIA embolisation during EVAR is provided in Table 1. The use of complex and expensive endovascular treatment technologies is necessary in order to keep blood flow to IIA (7). The development of new endovascular devices which would be simple in employment and allowing simultaneous treatment of both AAA and CIAA and preserving blood flow to IIA is therefore necessary. Available until recently endografts are based on exclusion of aneurysm from blood circulation and fixation in relatively healthy aortic tissues - that is in proximal and distal aneurysmal neck (14). However now there is a new generation sacsealing endograft with fixation performed in aneurysmal sac. Special polytetrafluoroethylene bags are filled by rapidly freezing polymer and blood circulation continues through two endografts tubes 'frozen' in the aneurysmal sac $(5,12)$. To the best of our knowledge until now there have been no publications comparing such new generation sac-sealing endograft function in patients after combined AAA and CIAA treatment.

\section{AIM OF THE STUDY}

The aim of the study was demonstrate successful treatment of AAA accompanied by CIAA preserving the blood flow in IIA using new generation sac sealing endovascular device.

\section{MATERIAL AND METHODS}

Since 2008 the Vascular Surgery Department of Pauls Stradins Clinical University Hospital (PSCUS) in Riga, Latvia is the largest clinical centre participating in the multicentre clinical trial of the new generation sacsealing endograft device (Nellix ${ }^{\circledR}$, Endologic, USA) displayed in Figure 4. Ethical Committee of PSCUS approval was obtained for performance of this clinical trial and all patients have signed an informed consent form. Patients were enrolled into the study if the morphology of their AAA and CIAA allowed treatment with Nellix endograft, that is AAA $>4.5 \mathrm{~cm}$ or aneurysmal growth rate exceeding $10 \%$ in one year, AAA neck $>$ $5 \mathrm{~mm}$ and diameter from $16-36 \mathrm{~mm}$, patent femoral and iliac arteries allowing endovascular repair. Patients with ruptured AAA, thoraco-abdominal aneurysms, allergies to contrast medium, occluded iliac and/or common femoral arteries and patients with creatinine level exceeding $2.0 \mathrm{mg} / \mathrm{dL}$ were excluded from the trial. During this period 40 patients were successfully treated using Nellix endografts. Endovascular procedures were performed in the hybrid operating theatre equipped with angiography device (Siemens). Patients were divided into two groups. In the treatment group 16 patients had simultaneous AAA and either unilateral or bilateral CIAA. The control group consisted of 24 patients with isolated AAA. Demographic patient data is provided in
Table 2. AAA diameter was $5.6 \pm 0.76 \mathrm{~cm}(\mathrm{~min}-4.3$ $\max -6.98)$ and $5.16 \pm 0.91 \mathrm{~cm}(\min -3.78$, max-7.24) in the treatment and control groups respectively. In the treatment group 7 patients had unilateral and 9 patients had bilateral CIAAs. CIAA diameter was $2.61 \pm 0.57 \mathrm{~cm}$ (min - 2.04, max - 4.44).

Patient follow-up was done at one, six and twelve months post-EVAR and on annual basis thereafter. General health condition, ankle-brachial index (ABI) and quality of life assessment was made during these post-procedural follow-up visits. The durability of Nellix endografts and blood flow in IIA was assessed using 64-layer CTA (General Electric LightSpeed) and DSU (Phillips iU22 xMatrix with multifrequence probe 2-4 MHz) performed by two experienced radiologists participating in the trial. Statistical analysis was done using SSPS software, v19.0 (IBM).

\section{RESULTS}

Long-term treatment results were prospectively analysed in patients after AAA and CIAA endovascular repair using new generation sac-sealing endograft device allowing successful CIAA treatment and preserving the blood flow to IIA. We assessed the efficacy and durability of the endograft, aneurysm related mortality and morbidity as well as post-procedural IIA patency. All procedures were performed under general anaesthesia and all patients remained in intensive care unit for the period of one day after the procedure. All patients were successfully treated with implanted endograft excluding AAA from blood circulation. In the treatment group all patients' CIAA were successfully treated and in all cases blood flow was preserved to IIA (Figure 2). Iliac extenders were used for the treatment of CIAA in eight cases of the treatment group. There have been no peri-operative deaths, myocardial infarctions or pelvic organs ischaemia in either treatment or control group. The length of procedure in the treatment group was $134 \pm 32 \mathrm{~min}$ and $104 \pm 28 \mathrm{~min}$ in control group. T-test provided statistically significant difference in procedural length between two groups $(\mathrm{p}<0.01)$. Average blood loss in the treatment group was $85 \mathrm{ml}$ (67.5-115) and $85 \mathrm{ml}$ (65-125) in the control group. Mann Whitney U test has not provided statistical difference between two groups $(p>0.05)$. The amount of polymer used in the treatment group and control group was $67.5 \mathrm{ml}$ (57-92) and $48 \mathrm{ml}$ (26.5-66) respectively. Average hospitalisation period was 7.5 days (4.5-10.5) and 5.5 days (4-6.5) in treatment and control groups respectively. Mann Whitney $U$ test showed statistically reliable difference between hospitalisation period length and amount of used polymer between two groups $(p<0.05)$. Length of procedure and amount of used polymer was larger in the treatment group due to the use of iliac extenders for CIAA treatment.

Average length of follow-up was 1.6 years (max - 4 years, min - 1 month). Neither of the patients have presented with clinical symptoms of pelvic organs' ischaemia, gluteal claudication or worsening in ABI. In all patients quality of life has returned back to preprocedural level after one month post-treatment (11). 
CTA and DUS follow-up in both group showed successfully excluded from blood circulation aneurysms (Figure 3). There have been no aneurysmal growth or endoleaks detected in either of the patients. All patients in both groups had patent IIA.

There have been two serious adverse events during the trial. In the treatment group on the 10th post-procedural month one patient with previously performed percutaneous transluminal coronary angioplasty (PTCA) died from myocardial infarction (MI). Another patient in the control group also with a history of PTCA has developed a MI with Q on the 15 th post-procedural day and was treated with PTCA and stent implantation. Both adverse events have developed notwithstanding aspirin and statins therapy. This has not resulted in mortality and morbidity differences between two groups.

\section{DISCUSSION}

Endovascular repair of AAA and CIAA has proved its effectiveness in the last decade $(9,14,23,25)$ and is used as a method of choice in numerous European and North American centres. The greatest advantage of this method is a relatively smaller patient trauma, reduced peri-operative morbidity and mortality in the early follow up period. However endovascular methods are limited by complex aneurysmal morphology (short and angulated aneurysmal neck, iliac artery aneurysms and tortuosity) and combination of AAA with CIAA (19, 27). Similarly, the results of our study showed $40 \%$ of patients with AAA extending to CIA.

Currently there are no approved devices allowing simultaneous treatment of AAA and CIAA. Usually the treatment in such cases is performed with combination of different endovascular devices or employing complex unapproved endograft solutions during clinical trials. Devices used outside the approved indications increase risks of associated complications as well as lower treatment durability. The development of currently available devices increases their indications for employment in patients with complex AAA morphology (8). Specifically this is important for patients with CIAA where the preservation of IIA patency is more difficult. According to literature data IIA occlusion is associated with up to $80 \%$ of gluteal claudication, $10 \%$ of impotence and $6-9 \%$ of intestinal ischaemia cases $(6$, $27)$. In the event of pelvic organs ischaemia there is a significant increase in procedure related morbidity and mortality $(15,17)$.

Preservation of IIA in younger patients is especially important in order to retain quality of life. The analysis of 550 patients in Farahmand et al study has provided that the biggest risk for development of gluteal claudication was where IIA embolisation was used for occlusion. The risk of endoleaks however was not reduced in this group, regardless of occluded IIA (7). In our study neither of the patients with CIAA developed any complications. One of the ways for preservation of IIA patency is combined endovascular and open repair where IIA is embolised during endovascular repair and thereafter reconstructed by open by-pass procedure from EIA or common femoral artery (CFA) to IIA (1). In this study performed by Arco et al two groups of patients were analysed first group received coil-embolisation of IIA before endovascular procedure and in other group IIA was covered with endograft and after patients was revascularised with open by-pass from EIA or CFA to IIA. Upon follow-up in the first group there were 21 patients with gluteal claudication and no complications observed in the revascularised group. The staged method applied in the second group somewhat contradicts the concept of non-invasive endovascular treatment where the procedure is performed through microincisions or completely percutaneously. This method also significantly increases the length and cost of the procedure.

Currently available alternative is endovascular treatment with branched endografts $(13,19)$. Also in this method there are limiting factors requiring CIAA diameter to be larger than 20-24 mm, without too tortuous IIA and EIA diameter of at least 6-8 $\mathrm{mm}$ with preferably no tortuosity. The requirement of such 'ideal' anatomy is necessary in order to implant a branched stent graft, however elderly patients and patients with large CIAA both IIA and EIA are usually significantly affected (10). As displayed by the latest studies with branched endografts it is possible to treat CIAA and preserve the blood flow to IIA, however this requires complex and expensive devices. This prolongs procedural time and decreases success rate of the procedure $(16,27)$. In addition to that, the increased complexity of the procedure reduces long-term outcome results. New generation sac-sealing endograft solution for the treatment of CIAA has provided that this device is not only effective but also simple in use and does not significantly increase the length of the procedure.

\section{CONCLUSIONS}

Our study shows successful treatment of AAA and simultaneous CIAA with new generation sac-sealing endograft. Eight cases required the use of iliac extenders to CIA, with the same simplicity in design and deployment, but only shorter in length. All patients had patent hypogastric artery on early and late follow-up. Serious adverse events during the trial in both treatment and control group were related to large prevalence of ischaemic heart disease in both groups. Further studies with larger number of patients and longer follow up period are necessary.

\section{Conflict of interest: None}

\section{REFERENCES}

1. Arko FR, Anthony LW, Bradley BH, Fogarthy TJ, Zarins CK. Hypogastric artery bypass to preserve pelvic circulation: Improved outcome after endovascular abdominal aortic aneurysm repair // J Vasc Surg, 2004; 39:404-8.

2. Bharwani N, Raja J, Choke E, Belli AM, Thompson MM, Morgan RA, Munneke G. Is internal iliac artery embolisation essential prior to endovascular repair of aortoiliac aneurysms? // Cardiovasc Intervent Radiol, 2008; 31:504-508. 
3. Bratby MJ, Munneke GM, Belli AM,Loosemore TM, Loftus I, Thompson MM, Morgan RA. How safe is bilateral iliac artery embolization prior to EVAR? // Cardiovasc Intervent Radiol 2008; 31:246-253.

4. Casey K, Al-Khatib WK, Zhou W. Hypogastric artery preservation during aortoiliac aneurysm repair // Annals of Vascular Surgery, 2011; 25;1:131-133.

5. Donayre CE, Zarins CK, Krievins D, Holden A, Hill A, Calderas C, Velez J, White RA. Initial clinical experience with a sac-anchoring endoprosthesis for aortic aneurysm repair // J Vasc Surg, 2011; 53(3):574-582.

6. Engelke C, Elford J, Morgan RA, Belli AM. Internal iliac artery embolization with bilateral occlusion before endovascular aortoiliac aneurysm repair clinical outcome of simultaneous and sequential intervention // J Vasc Interv Radiol, 2002; 13:667676.

7. Farahmand P, Becquemin JP, Desgranges P, Allaire E, Marzelle J, Roudot-Thoraval F.Is hypogastric artery embolization during endovascular aortoiliac aneurysm repair (EVAR) innocuous and useful? // Eur J Vasc Endovasc Surg, 2008;35:429-435.

8. Goncalves FB, Vries JPPM, Keulen JW, Dekker H, Moll FL, Herwaarden JA, Verhagen HJM. Severe proximal aneurysm neck angulation: early results using the endurant stentgraft system // Eur J Vasc Endovasc Surg, 2011; 41:193-200.

9. Greenhalgh RM, Brown LC, Kwong GP, Powell JT, Thompson SG; EVAR trial participants. Comparison of endovascular aneurysm repair with open repair in patients with abdominal aortic aneurysm (EVAR trial 1), 30-day operative mortality results: randomised controlled trial // Lancet, 2004; 364(9437):843-848.

10. Karthikesalingam A, Hinchliffe RJ, Holt PJE, Boyle JR, Loftus IM, Thompson MM. Endovascular aneurysm repair with preservation of internal iliac artery using the iliac branch graft device // Eur J Vasc Endovasc Surg, 2010; 39:285-294.

11. Kisis K, Krievins D, Naskovica K, Gedins M, Savlovskis J, Ezite N, Lietuvietis E, Zarins K. Quality of life after endovascular abdominal aortic aneurysm repair: Nellix sac-anchoring endoprosthesis versus open repair // Medicina, 2012; 48(6):286-291.

12. Krievins D, Holden A, Savlovskis J, Calderas C, Donayre CE, Moll FL, Katzen B, Zarins CK. EVAR using the Nellix Sac-anchoring endoprosthesis: treatment of favourable and adverse anatomy // Eur J Vasc Endovasc Surg, 201 1; 42(1):38-46.

13. Lee WA. Branched endograft for aortoiliac artery aneurysms // Vascular, 2009; 17(3):111-118.

14. Malas MB, Julie AF. Interpretation of the resultas of OVER in context of EVAR trial, DREAM and the EUROSTAR registry // Semin Vasc Surg, 2010; 23:165-169.

15. Maldonado TS, Rockman CB, Riles E, Douglas D, Adelman MA, Jacobowitz GR, Riles TS. Ischemic complications after endovascular abdominal aortic aneurysm repair // J Vasc Surg, 2004; 40:703-710.
16. Malina M, Dirven M, Sonesson B, Resch T, Dias N, Ivancev K. Feasibility of a branched stent-graft in common iliac artery aneurysms // J Endovasc Ther, 2006; 13:496-500.

17. Miller A, Marota M, Scordi-Bello I, Tammaro Y, Marin M, Divino C. Ischemic colitis after endovascular aortoiliac aneurysm repair // Arch Surg, 2009; 144(10):900-903.

18. Moll FL et al. Managment of Abdominal Aortic Aneurysms clinical practice guaidlines of the European society for vascular surgery // Eur J Vasc Endovasc Surg, 2011; 41:1-58.

19. Oderich GS, Greenberg RK. Endovascular iliac branch devices for iliac aneurysms // Perspectives in Vascular Surgery and Endovascular Therapy, $2011 ; 23(3): 166-172$.

20. Parodi JC, Parodi FE. The sandwich technique to preserve the hypogastric artery during EVAR // J Endovasc Ther, 2011; 18:112-113.

21. Pavlidis D, Hormann M, Libicher M, Gawenda M, Brunkwall J. Buttock claudication after interventional occlusion oft he hypogastric artery a mid-term follow-up // Vascular and Endovascular Surgery, 2012; 46(3):236-241.

22. Pepellenbosch N, CuypersWM, Vahl AC, Vermassen F, Buth J. Emergency endovascular treatment for ruptured abdominal aortic aneurysms and the risk of spinal cord ischemia // J Vasc Surg, 2005; 42:608614.

23. United Kingdom EVAR Trial investigators, Greenhalgh RM, Brown LC, et al. Endovascular versus open repair of abdominal aortic aneurysm // N Engl J Med, 2010; 362(20):1863-1871.

24. Rayt HS, Bown MJ, Lambert KV, Fishwick NG, McCarthy MJ, London NJM, Sayers RD. Buttock claudication and erectile dysfunction after internal iliac artery embolization in patients prior to endovascular aortic aneurysm repair // Cardiovasc Intervent Radiol, 2008; 31:728-734.

25. Stroupe KT, Lederle FA, Matsumura JS, et al. Costeffectiveness of open versus endovascular repair of abdominal aortic aneurysm in the OVER trial // J Vasc Surg, 2012; 56(4):901-909.

26. Tefera G, Turnipsed WD, Carr SC, Pulfer KA, Hoch JR, Acher CW. Is coil embolisation of hypogastric artery necessary during endovascular treatment of aortoiliac aneurysms? // Annals of Vascular Surgery, 2004; 18(2):143-146.

27. Verzini F, Parlani G, Romano L, De Rango P, Panuccio G, Piergiorgio C. Endovascular treatment of iliac aneurysms: concurrent comparison of side branch endograft versus hypogastric exclusion // J Vasc Surg, 2009; 49(5):154-1161.

28. Zander $T$, Baldi $S$, Rabellino $M$, Rostagno $R$, Isaza B, Llorens R, Carreira JM, Maynar M. Bilateral hypogastric artery occlusion in endovascular repair of abdominal aortic aneurysms and its clinical significance // J Vasc Interv Radiol, 2007; 18:14811486. 
Table 1. Incidence of complications after unilateral or bilateral IIA coil embolisation prior to EVAR

\begin{tabular}{|l|c|c|c|c|}
\hline Author/year & $\begin{array}{c}\text { Number of } \\
\text { Patients }\end{array}$ & Gluteal Claudication & $\begin{array}{c}\text { Spinal Cord } \\
\text { Ischaemia }\end{array}$ & $\begin{array}{c}\text { Erectile } \\
\text { Dysfunction }\end{array}$ \\
\hline Arko et al, 2004 & 12 & $6 / 12(50 \%)$ & - & - \\
\hline Bratby et al, 2008 & 39 & $12 / 39(31 \%)$ & $1 / 39(2 \%)$ & $2 / 37(5 \%)$ \\
\hline Engelke et al, 2002 & 16 & $4 / 16(25 \%)$ & - & $1 / 16(6 \%)$ \\
\hline Farahmand et al, 2008 & 76 & $44 / 76(58 \%)$ & - & $15 / 76(21 \%)$ \\
\hline Pavlidis et al, 2012 & 39 & $20 / 39(51 \%)$ & - & - \\
\hline Rayt et al, 2008 & 29 & $16 / 29(55 \%)$ & - & - \\
\hline Tefera et al, 2004 & 13 & $7 / 13(53 \%)$ & - & - \\
\hline Zander et al, 2007 & 14 & $4 / 14(29 \%)$ & - & $1 / 14(7 \%)$ \\
\hline
\end{tabular}

Table 2. Demographic data in treatment and control groups

\begin{tabular}{|c|c|c|}
\hline & Treatment Group & Control Group \\
\hline Male & 15 & 15 \\
\hline Female & 1 & 9 \\
\hline Average age at the time of procedure (years) & $70 \pm 8.20(53-83)$ & $70 \pm 6.99(58-86)$ \\
\hline Coronary artery disease & $10(63 \%)$ & $8(33 \%)$ \\
\hline Peripheral artery disease & $3(19 \%)$ & $5(21 \%)$ \\
\hline Hypertension & $8(50 \%)$ & $12(50 \%)$ \\
\hline PTCA or CABG & $6(38 \%)$ & $4(17 \%)$ \\
\hline Diabetus mielitus & $1(6 \%)$ & - \\
\hline History of intra-abdominal surgery & $4(25 \%)$ & $1(4 \%)$ \\
\hline
\end{tabular}

\section{Address:}

Dr Kaspars Kisis

Department of Vascular Surgery

Pauls Stradins Clinical University Hospital

13 Pilsonu Street, LV-1002, Riga, Latvia

Email: kaspars.kisis@stradini.lv 


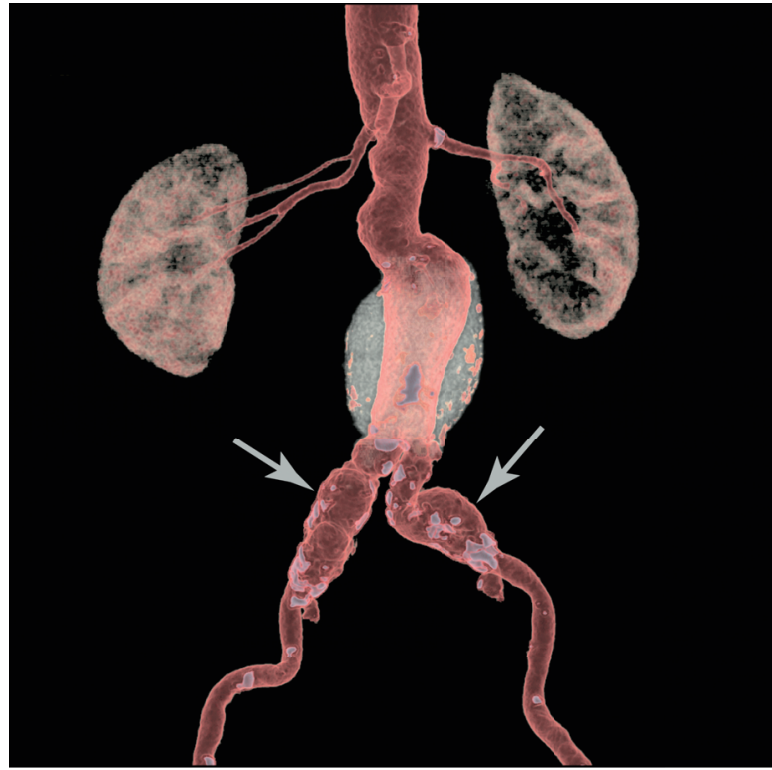

Fig. 1. CTA reconstruction in a patient with AAA combined with bilateral CIAA (arrows)

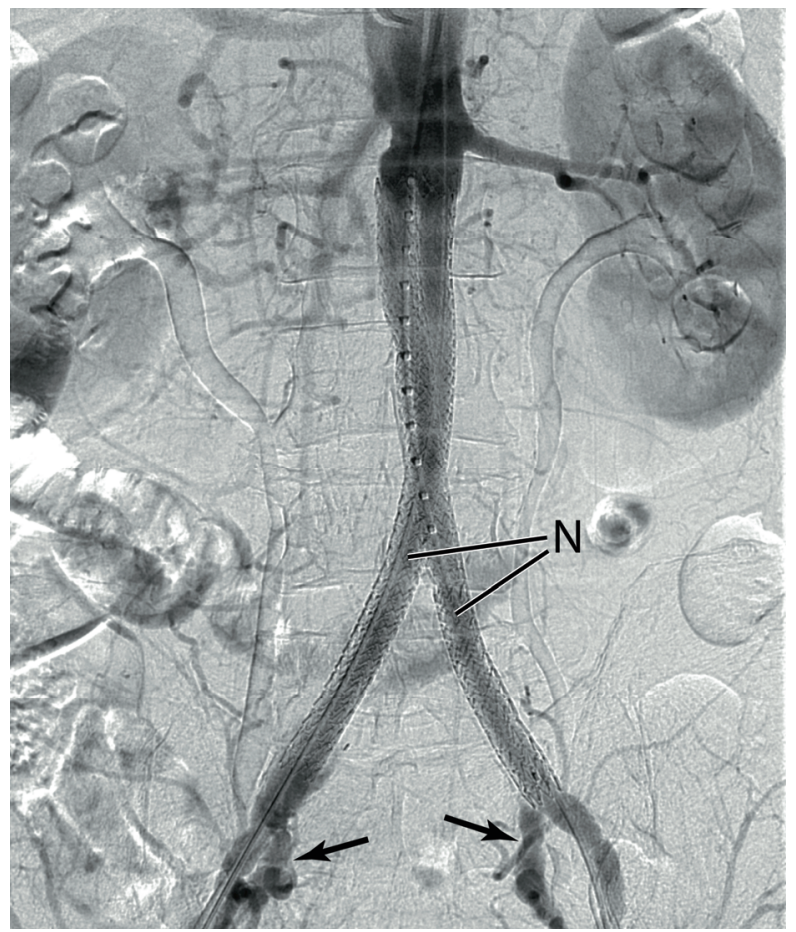

Fig. 2. Control DSA immediately after deployment of new generation sac-sealing endograft: AAA and both CIAA excluded from circulation, stent graft tubes frozen inside aneurysmal sac provide the blood flow continuance through endograft $(\mathrm{N})$ to both patent IIA (arrows)

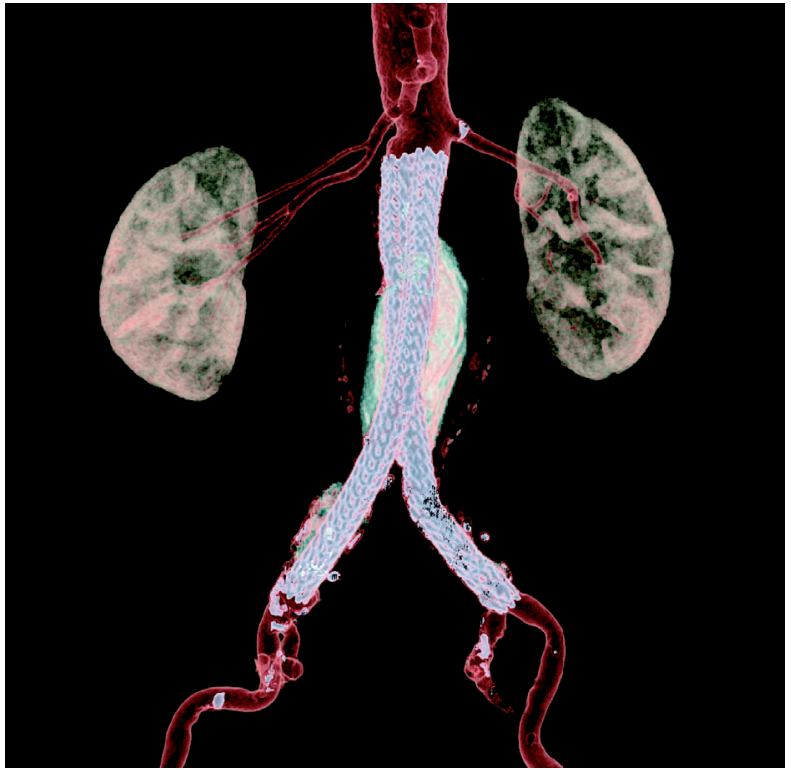

Fig. 3. CTA reconstruction in patient 3 years after procedure. Aneurysms excluded from circulation. Endograft is stable and fixed in the aneurysmal sac, blood flow preserved to IIAs

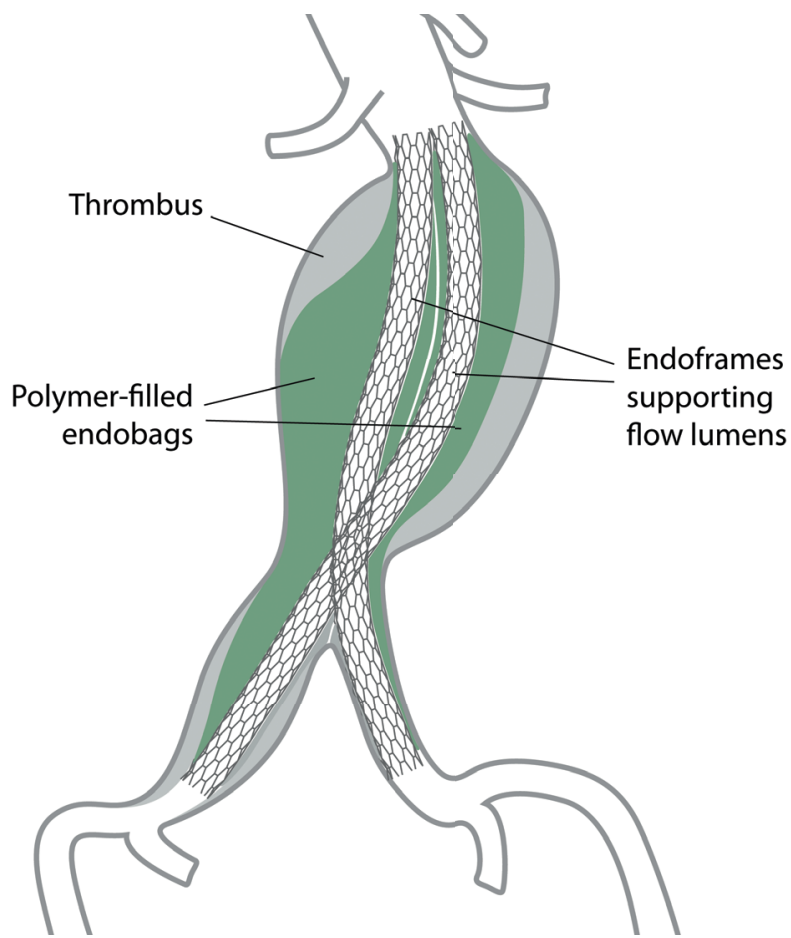

Fig. 4. Design of new generation sac-sealing endograft device (Nellix) 